\title{
Necessidades e Preocupações Maternas no Período Pós-Parto: Revisão Sistemática da Literatura
}

\begin{abstract}
Regina Rasteiro', Eduardo Santos ${ }^{1}$ e Emília Coutinho ${ }^{2}$
${ }^{1}$ Centro Hospitalar e Universitário de Coimbra, Portugal | regina.rasteiro@gmail.com; ejsantos@gmail.com | https://orcid.org/0000-0003-0797-349X; https://orcid.org/0000-00030557-2377

${ }^{2}$ Instituto Politécnico de Viseu, Portugal | ecoutinhoessv@gmail.com | https://orcid.org/0000 0002-9506-4626

Resumo: No período pós-parto, as mães, adaptando-se ao seu novo papel, confrontam-se com necessidades e preocupacões que influenciam o modo como vivenciam esta fase. Objetivo: identificar necessidades e preocupações maternas, no primeiro ano pós-parto, contribuindo para práticas de enfermagem sustentadas. Métodos: Revisão sistemática da literatura. A pesquisa, realizada na ESBCOhost, Web of Science, Scopus, PubMed, OAlster e RCAAP, entre 2016-2020, texto integral, em português/inglês/espanhol, incluiu 20 artigos cuja qualidade metodológica foi avaliada através do Critical Apraisal Skills Programme ou Mixed Methods Appraisal Tool. Resultados: Emergiram seis categorias de necessidades maternas, e preocupações, nomeadamente a necessidade de recuperação física e psicológica, de desenvolver habilidades e confiança para cuidar do recém-nascido, a necessidade de informação, de uma vida conjugal e sexual satisfatórias, de apoio familiar e social e dos profissionais de saúde. Conclusões: O/A Enfermeiro/a Especialista em saúde materna e obstétrica deve diagnosticar as preocupações maternas e construir um plano de cuidados personalizado, centrado na mulher/casal/família, para lhes dar resposta, reforçando a competência materna. Sugere-se o desenvolvimento de programas de recuperação pósparto e a realização de visitas domiciliárias para atender às necessidades maternas.
\end{abstract}

Palavras-chave: Período Pós-Parto; Parentalidade; Papel do Profissional de Enfermagem; Necessidades e Demandas de Serviços de Saúde.

\section{Maternal Needs and Concerns in Postpartum Period: Systematic Review}

\begin{abstract}
In postpartum period, mothers are confronted with needs and concerns that influence the way they experience this phase while adapting to their new role. Objective: to identify maternal needs and concerns, in the first postpartum year, contributing to sustained nursing practices. Methods: Systematic literature review. The research, carried out at ESBCOhost, Web of Science, Scopus, PubMed, OAlster and RCAAP, between 2016-2020, full text, in Portuguese/English/Spanish, included 20 articles whose methodological quality was assessed through the Critical Apraisal Skills Program or Mixed Methods Appraisal Tool. Results: Six categories of maternal needs emerged, namely the need for physical and psychological recovery, to develop skills and confidence to care for the newborn, the need for information, for a satisfactory conjugal and sexual life, for family and social support and health professionals' support. Conclusions: Midwives must diagnose maternal concerns and build a personalized care plan, centered on woman/couple/family, to respond to them, reinforcing maternal competence. It is suggested the development of postpartum recovery programs and home visits to meet maternal needs.
\end{abstract}

Keywords: Postpartum Period; Parenting; Nurse's Role; Health Services Needs and Demands.

\section{Introdução}

O período pós-parto (PPP) é ímpar na vida da mulher. Esta adapta-se às mudanças que em si se vão operando, ao mesmo tempo que aprende a lidar com o filho, a acolhê-lo na sua vida, em família, e na sociedade.

Mercer (2004) desenvolveu a teoria da Consecução do Papel Maternal, operacionalizada em quatro fases, uma com foco na gravidez e três no PPP, sem limites temporais rígidos. A primeira fase do PPP caracteriza-se pela busca do conhecimento e pela necessidade de aprendizagem, relativamente ao filho, ao mesmo tempo que o seu corpo recupera do parto. 
Na segunda fase, "seguindo em direção a um novo normal", há um reajustamento no modo como vive as suas relações (conjugal, familiar e entre amigos), ao mesmo tempo que sente um aumento de confiança para cuidar do filho. Na última, a mãe já assume uma identidade materna, sentindo-se competente nas suas atividades, em cuidar de si e do filho (Mercer, 2004).

No PPP, as exigências com o filho deixam as mães ansiosas e preocupadas, por quererem cumprir adequadamente o seu papel (American College of Obstetricians and Gynecologists, 2018). Almalik (2017) refere que as suas preocupações nem sempre são completamente esclarecidas e isso, segundo Negron et al. (2013), pode levar a sentimentos de frustração e depressão.

A pertinência desta revisão sistemática da literatura (RSL) de abordagem qualitativa, realizada no âmbito da ciência de Enfermagem, prende-se com o facto permitir ao/à Enfermeiro/a Especialista em enfermagem de saúde materna e obstétrica (EEESMO) prever as necessidades maternas e definir estratégias que reforcem a competência materna, apoiando no processo de transição para a parentalidade, competência definida pela Ordem dos Enfermeiros (2019).

Para dar resposta à pergunta PICo: "Quais as preocupações e necessidades maternas no período pós-parto, após a alta hospitalar?", as participantes (P) são as mães ou puérperas, o fenómeno de interesse (I) a estudar são as preocupações e necessidades maternas e o contexto (Co) é o do pós-parto, no domicílio.

O objetivo é identificar as preocupações e as necessidades maternas, após a alta hospitalar, no domicílio, no primeiro ano pós-parto.

\section{Metodologia}

Realizou-se uma pesquisa prévia à elaboração desta RSL, para verificar a pertinência do tema, evitando a duplicação de estudos. O seu protocolo foi submetido no PROSPERO (International prospective register of systematic reviews), com 0 registo CRD42020203838, disponível em: https://www.crd.york.ac.uk/prospero/display_record.php?RecordID=203838.

A revisão seguiu a metodologia proposta pela JBI (Aromataris \& Munn, 2020). Os descritores utilizados foram confirmados como termos MeSH (medical subject Heading) no site www.ncbi.nlm.nih.gov/mesh. Na ausência de confirmação foi utilizada a correspondente linguagem natural. Quando as bases de dados utilizavam outros léxicos de indexação, os descritores e linguagem natural foram adaptados.

A pesquisa realizou-se de 20 de outubro a 12 de novembro de 2020, na Pubmed, EBSCOhost, Web of Science e Scopus. Na tentativa de consultar toda a evidência existente para reduzir o viés dos resultados, pesquisou-se alguma literatura cinzenta, na OAlster e no Repositório Científico de Acesso Aberto em Portugal (RCAAP).

Usou-se a fórmula de pesquisa: \#1 descritor Mesh postpartum women OR mother OR maternal), \#2 needs OR concerns; \#3 descritor Mesh postpartum period OR postnatal OR puerperium; \#4 [\#1 AND \#2 AND \#3] (título/resumo).

Para identificar os estudos a incluir na RSL, que contribuíssem para a prática portuguesa de enfermagem, foram definidos critérios de inclusão e de exclusão (tabela 1).

A pesquisa foi limitada aos últimos 5 anos, para obter as mais atuais vivências maternas, num período de facilidade de acesso a informação e excluíram-se os artigos desenvolvidos em países pertencente à lista das Nações Unidas dos menos desenvolvidos (least developed countries - LDC), em que o acesso e nível de saúde, as condições de vida e aspetos culturais diferem de Portugal, o que enviesaria os resultados. 
Vol. 8 | Investigação Qualitativa em Saúde: Avanços e Desafios

Tabela 1. Critérios de inclusão e de exclusão

\begin{tabular}{lll}
$\begin{array}{l}\text { Critérios de } \\
\text { seleção }\end{array}$ & \multicolumn{1}{c}{ Critérios de inclusão } & \multicolumn{1}{c}{ Critérios de exclusão } \\
\hline Participantes & Mães de crianças saudáveis & Estudos apenas em pais (e não mães) \\
& $\begin{array}{l}\text { Sem patologias crónicas ou depressão } \\
\text { pós-parto; sem problemas de abuso de } \\
\text { substâncias }\end{array}$ & Mães que sofreram morte fetal \\
& & Mães de filhos com problemas de saúde \\
Fenómeno & Preocupações e necessidades maternas & Mães com problemas de abuso de \\
de Interesse & substâncias & necessidades maternas \\
& Durante o primeiro ano pós-parto & Mães ou filhos em regime de internamento \\
& Após a alta hospitalar, no domicílio & Apenas abordar a gravidez
\end{tabular}

Para avaliar a qualidade metodológica, aplicou-se o Critical Appraisal Skills Programme (CASP) em estudos qualitativos (10 perguntas) e, em estudos mistos, o Mixed Methods Appraisal Tools (MMAT) (duas perguntas iniciais, seguidas de 15).

A qualidade metodológica de cada estudo é cotada como baixa entre zero a $50 \%$ (inclusive) de respostas positivas, qualidade moderada entre 50 e $85 \%$ (inclusive) e alta em valores superiores a $85 \%$.

A seleção dos estudos, a extração e síntese de dados (usando o instrumento JBI-QARI data extraction tool) foi realizada por dois investigadores. Na ausência de consenso, foi incluído um terceiro (critério de desempate).

\section{Resultados}

O processo de seleção dos estudos incluídos está explanado na figura 1.

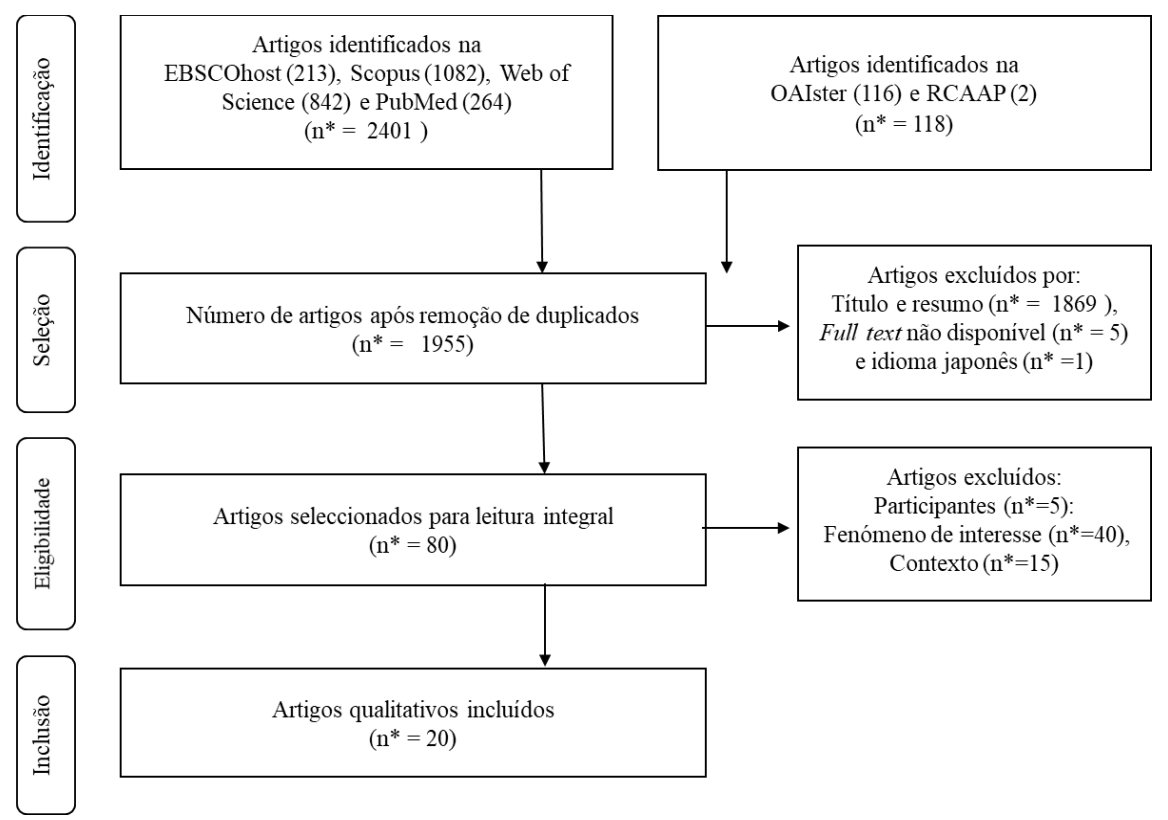

Fig. 1. Diagrama de fluxo de acordo com o PRISMA. 
Os estudos incluídos são de cariz qualitativo, à exceção do de Rouhi et al. (2019), que é misto. Na tabela 2, é realizada a análise dos mesmos.

Tabela 2: Síntese dos artigos na RSL

\begin{tabular}{|c|c|c|c|}
\hline Autores, data e país & $\begin{array}{l}{ }^{\star} \text { Participantes } \\
{ }^{\star \star} \text { Objetivos }\end{array}$ & Principais resultados & $\begin{array}{c}\text { Avaliação da } \\
\text { qualidade } \\
\text { metodológica }\end{array}$ \\
\hline Albanese et al. (2020), & *30 puérperas & A forma como a mãe vive o PPP & $100 \%$ Alta \\
\hline Austrália & $\begin{array}{l}{ }^{* *} \text { Identificar fatores } \\
\text { promotores de bem-estar } \\
\text { materno. }\end{array}$ & $\begin{array}{l}\text { influencia a sua saúde e a dos filhos. É } \\
\text { necessária uma abordagem materna } \\
\text { individual e visitas domiciliárias (VD). }\end{array}$ & \\
\hline $\begin{array}{l}\text { Farewell et al. (2020), } \\
\text { EUA }\end{array}$ & $\begin{array}{l}\text { *27 mulheres ( } 48 \% \text { nos } \\
\text { primeiros } 6 \text { meses pós- } \\
\text { parto, as restantes são } \\
\text { grávidas) }\end{array}$ & $\begin{array}{l}\text { Lidar com o stress, depressão e } \\
\text { ansiedade são desafios das puérperas, } \\
\text { em plena pandemia. A necessidade de } \\
\text { isolamento social agrava necessidades }\end{array}$ & $93,3 \%$ Alta \\
\hline & $\begin{array}{l}{ }^{* *} \text { Compreender o estado } \\
\text { de saúde mental } \\
\text { materno, durante a } \\
\text { pandemia. }\end{array}$ & $\begin{array}{l}\text { de suporte e de comportamentos de } \\
\text { coping. }\end{array}$ & \\
\hline $\begin{array}{l}\text { Faria-Schützer et al. } \\
(2020) \text {, Brasil }\end{array}$ & $\begin{array}{l}{ }^{*} 16 \text { mulheres obesas } \\
{ }^{* \star} \text { Explorar as } \\
\text { experiências relativas ao } \\
\text { autocuidado e apoio } \\
\text { recebido. }\end{array}$ & $\begin{array}{l}\text { O foco das puérperas são os filhos. Os } \\
\text { profissionais de saúde devem ter } \\
\text { atenção por estas mulheres, por } \\
\text { descurarem a sua saúde, } \\
\text { nomeadamente a alimentação. }\end{array}$ & $90 \%$ Alta \\
\hline $\begin{array}{l}\text { Marambaia et al. (2020), } \\
\text { Brasil }\end{array}$ & $\begin{array}{l}{ }^{*} 12 \text { puérperas, } \\
\text { submetidas a } \\
\text { episiotomia } \\
{ }^{* *} \text { Analisar a vivência } \\
\text { sexualidade. }\end{array}$ & $\begin{array}{l}\text { O medo da dor ao reiniciar a atividade } \\
\text { sexual, insatisfação com a sua imagem } \\
\text { corporal têm um impacto negativo na } \\
\text { vivência da sexualidade, no PPP. }\end{array}$ & $100 \%$ Alta \\
\hline Nan et al. (2020), China & $\begin{array}{l}\text { *28 puérperas } \\
\text { **Explorar o ponto de vista } \\
\text { materno relativamente aos } \\
\text { cuidados prestados pelos } \\
\text { serviços no PPP, } \\
\text { identificar barreira à sua } \\
\text { utilização. }\end{array}$ & $\begin{array}{l}\text { As mães sentem insatisfação com o } \\
\text { atendimento que têm no PPP, relutância } \\
\text { a recorrer aos profissionais de saúde e } \\
\text { referem que os cuidados deviam } \\
\text { combinar métodos presenciais com } \\
\text { online. }\end{array}$ & $100 \%$ Alta \\
\hline $\begin{array}{l}\text { Xiao, Loke et al. (2020), } \\
\text { China }\end{array}$ & $\begin{array}{l}{ }^{*} 22 \text { mulheres nas } \\
\text { primeiras } 6 \text { semanas } \\
\text { pós-parto } \\
{ }^{* \star} \text { Compreender as } \\
\text { experiências maternas } \\
\text { com a amamentação e } \\
\text { as necessidades de } \\
\text { apoio. }\end{array}$ & $\begin{array}{l}\text { Um ambiente de suporte é fundamental } \\
\text { para manter a amamentação. O apoio } \\
\text { dos maridos, mães e sogras influencia a } \\
\text { vivência deste período, pelo que } \\
\text { também devem ser alvo de ensinos. }\end{array}$ & $100 \%$ Alta \\
\hline $\begin{array}{l}\text { Xiao, Ngai et al. (2020), } \\
\text { China }\end{array}$ & $\begin{array}{l}{ }^{\star} 22 \text { puérperas no } 30^{\circ} \text { e } \\
42^{\circ} \text { dias pós-parto } \\
{ }^{\star \star} \text { Explorar as } \\
\text { dificuldades, } \\
\text { necessidades e as } \\
\text { preocupações nas } \\
\text { primeiras seis semanas } \\
\text { pós-parto. }\end{array}$ & $\begin{array}{l}\text { As preocupações relacionam-se com a } \\
\text { recuperação materna e a necessidade } \\
\text { de serem reconhecidas como mães } \\
\text { competentes. Os profissionais de saúde } \\
\text { devem considerar a família como um } \\
\text { todo. }\end{array}$ & $100 \%$ Alta \\
\hline $\begin{array}{l}\text { Olander et al. (2019), } \\
\text { Inglaterra }\end{array}$ & $\begin{array}{l}{ }^{*} 29 \text { mães } \\
\text { **Explorar experiências } \\
\text { maternas sobre } \\
\text { continuidade e partilha } \\
\text { da informação entre } \\
\text { profissionais. }\end{array}$ & $\begin{array}{l}\text { As mães sentem-se ignoradas, que há } \\
\text { pouca partilha de informação entre } \\
\text { profissionais e prestação de } \\
\text { informações inconsistentes. Sugerem } \\
\text { visitas da equipa de obstetrícia e } \\
\text { pediatria em simultâneo, evitando } \\
\text { incongruências nas orientações. }\end{array}$ & $90 \%$ Alta \\
\hline
\end{tabular}




\begin{tabular}{|c|c|c|c|}
\hline Autores, data e país & $\begin{array}{l}\text { *Participantes } \\
{ }^{\star \star} \text { Objetivos }\end{array}$ & Principais resultados & $\begin{array}{c}\text { Avaliação da } \\
\text { qualidade } \\
\text { metodológica }\end{array}$ \\
\hline $\begin{array}{l}\text { Rouhi et al. (2019), } \\
\text { Austrália }\end{array}$ & $\begin{array}{l}{ }^{*} 81 \text { mães } \\
{ }^{* \star} \text { Identificar } \\
\text { necessidades maternas } \\
\text { e comportamentos de } \\
\text { procura de ajuda } \\
\text { associados. }\end{array}$ & $\begin{array}{l}\text { Há necessidades de apoio social, } \\
\text { familiar, de saúde e fitness. Os } \\
\text { problemas pós-parto são mais amplos } \\
\text { para as mulheres, do que para os } \\
\text { profissionais, que só se focam no RN. }\end{array}$ & $93,3 \%$ Alta \\
\hline $\begin{array}{l}\text { Shorey \& Ng (2019), } \\
\text { Singapura }\end{array}$ & $\begin{array}{l}\text { *20 puérperas, em risco } \\
\text { de depressão } \\
{ }^{* *} \text { Avaliar as } \\
\text { experiências maternas } \\
\text { sobre um programa de } \\
\text { apoio de pares }\end{array}$ & $\begin{array}{l}\text { As mães mostram-se satisfeitas, não se } \\
\text { sentem tão sozinhas e têm menos } \\
\text { sentimentos negativos. Este tipo de } \\
\text { apoio promove o bem-estar materno no } \\
\text { PPP, mesmo em mães mais } \\
\text { vulneráveis. }\end{array}$ & $100 \%$ Alta \\
\hline $\begin{array}{l}\text { Yamada et al. (2019), } \\
\text { EUA }\end{array}$ & $\begin{array}{l}{ }^{*} \text { Puérperas de um grupo } \\
\text { online que colocaram } \\
543 \text { posts } \\
{ }^{* *} \text { Categorizar as } \\
\text { questões maternas, num } \\
\text { fórum online, acerca do } \\
\text { leite materno extraído } \\
\text { por bomba }\end{array}$ & $\begin{array}{l}\text { As preocupações maternas são a } \\
\text { quantidade de leite adequada a extrair } \\
\text { mecanicamente e a oferecer aos filhos. } \\
\text { É necessário apoio para promover a } \\
\text { continuidade do aleitamento. }\end{array}$ & $90 \%$ Alta \\
\hline $\begin{array}{l}\text { Henshaw et al. (2018), } \\
\text { EUA }\end{array}$ & $\begin{array}{l}{ }^{*} 26 \text { mães, } 6 \text { pais } \\
{ }^{* *} \text { Explorar as } \\
\text { experiências de procura } \\
\text { de informação, nas } \\
\text { primeiras seis semanas. }\end{array}$ & $\begin{array}{l}\text { Pais e mães sentem-se desamparados, } \\
\text { assoberbados com a quantidade de } \\
\text { informação disponível, necessitando de } \\
\text { ensino credível e suporte seguro. }\end{array}$ & $100 \%$ Alta \\
\hline Zhang et al. (2018), China & $\begin{array}{l}\text { *10 mães que } \\
\text { amamentaram } \\
{ }^{\star \star} \text { Explorar as } \\
\text { experiências com a } \\
\text { amamentação } \\
\text { (dificuldades e } \\
\text { necessidades de apoio) }\end{array}$ & $\begin{array}{l}\text { As necessidades relativas à } \\
\text { amamentação diferem no tempo. Após } \\
6 \text { meses, amamentar em público gera } \\
\text { embaraço, vontade de suspender a } \\
\text { amamentação. Deve encorajar-se a } \\
\text { amamentação exclusiva até aos } 6 \\
\text { meses e a prolonga-la até aos } 2 \text { anos. }\end{array}$ & $100 \%$ Alta \\
\hline $\begin{array}{l}\text { Carvalho et al. (2017), } \\
\text { Portugal }\end{array}$ & $\begin{array}{l}\text { *11 primíparas } \\
{ }^{* *} \text { Identificar as principais } \\
\text { dificuldades de } \\
\text { primíparas, no primeiro } \\
\text { semestre pós-parto. }\end{array}$ & $\begin{array}{l}\text { As principais dificuldades são a } \\
\text { recuperação pós-parto, cuidar do } \\
\text { recém-nascido (RN) e a relação } \\
\text { conjugal. Estas influenciam } \\
\text { negativamente a parentalidade. Os } \\
\text { enfermeiros devem ser sensíveis às } \\
\text { necessidades das mães e famílias. }\end{array}$ & $100 \%$ Alta \\
\hline Corrêa et al. (2017), Brasil & $\begin{array}{l}{ }^{*} 10 \text { mulheres } \\
{ }^{\star \star} \text { Compreender as } \\
\text { práticas profissionais e } \\
\text { as perceções maternas } \\
\text { inerentes. }\end{array}$ & $\begin{array}{l}\text { As mulheres sentem-se insatisfeitas } \\
\text { com a VD por ser tardia, na ausência de } \\
\text { médico/enfermeiro, priorizando o RN. A } \\
\text { visita e a consulta puerperal devem ser } \\
\text { adaptadas às necessidades maternas. }\end{array}$ & $100 \%$ Alta \\
\hline $\begin{array}{l}\text { Masala-Chokwe \& } \\
\text { Ramukumba (2017), } \\
\text { África do Sul }\end{array}$ & $\begin{array}{l}\text { *14 primíparas (com alta } \\
\text { precoce) } \\
{ }^{\star \star} \text { Compreender o } \\
\text { significado atribuído à } \\
\text { alta precoce e às } \\
\text { necessidades sentidas. }\end{array}$ & $\begin{array}{l}\text { As mães sentiram falta de apoio social, } \\
\text { de confiança, de conhecimentos e } \\
\text { habilidades para cuidar do RN e delas } \\
\text { próprias. É necessário identificar tipos } \\
\text { de suporte alternativos, para promover } \\
\text { o ajustamento à maternidade. }\end{array}$ & $100 \%$ Alta \\
\hline $\begin{array}{l}\text { Slomian et al. (2017), } \\
\text { Bélgica }\end{array}$ & $\begin{array}{l}{ }^{*} 22 \text { puérperas com e } \\
\text { sem sintomas } \\
\text { depressivos } \\
{ }^{\star \star} \text { Explorar as } \\
\text { necessidades sentidas } \\
\text { pelas mães e comparar } \\
\text { com as perceções dos } \\
\text { pais e profissionais de } \\
\text { saúde }\end{array}$ & $\begin{array}{l}\text { Há necessidade de informação, de } \\
\text { apoio psicológico e prático. As } \\
\text { perceções de mães, pais e profissionais } \\
\text { diferem. As mães sentem necessidades } \\
\text { semelhantes. Tabus sobre dificuldades } \\
\text { maternas impede as mães de procurar } \\
\text { apoio quando necessitam. }\end{array}$ & $100 \%$ Alta \\
\hline
\end{tabular}




\begin{tabular}{|c|c|c|c|}
\hline Autores, data e país & $\begin{array}{l}{ }^{\star} \text { Participantes } \\
{ }^{\star \star} \text { Objetivos }\end{array}$ & Principais resultados & $\begin{array}{c}\text { Avaliação da } \\
\text { qualidade } \\
\text { metodológica }\end{array}$ \\
\hline $\begin{array}{l}\text { Cantarino et al. (2016), } \\
\text { Espanha }\end{array}$ & $\begin{array}{l}{ }^{\star} 50 \text { puérperas } \\
\text { espanholas e } 20 \\
\text { imigrantes } \\
{ }^{* *} \text { Conhecer as vivências } \\
\text { da sexualidade no PPP e } \\
\text { distinguir diferenças } \\
\text { entre as de espanholas e } \\
\text { as de imigrantes. }\end{array}$ & $\begin{array}{l}\text { O retomar das relações sexuais está } \\
\text { associado a medo e dor. As mulheres } \\
\text { espanholas retomam mais cedo que as } \\
\text { imigrantes, procurando ajuda nas } \\
\text { mulheres da família. As imigrantes, por } \\
\text { questões culturais, não abordam o tema } \\
\text { da sexualidade, associando-a a } \\
\text { procriação e não a prazer, mas ambas } \\
\text { necessitam de ajuda e compreensão } \\
\text { dos profissionais de saúde. }\end{array}$ & $100 \%$ Alta \\
\hline Kurth et al. (2016), Suíça & $\begin{array}{l}{ }^{*} 20 \text { mães e } 4 \text { pais } \\
{ }^{* *} \text { Analisar as } \\
\text { experiências dos novos } \\
\text { pais relativamente aos } \\
\text { cuidados prestados, } \\
\text { após uma alta precoce. }\end{array}$ & $\begin{array}{l}\text { As necessidades maternas e do RN } \\
\text { fazem com que precisem de apoio. Há } \\
\text { dificuldade no acesso a cuidados pós- } \\
\text { parto (que são fragmentados) e há falta } \\
\text { de coordenação profissional. Sugerem } \\
\text { VD ou linhas de ajuda para atender às } \\
\text { necessidades. }\end{array}$ & $100 \%$ Alta \\
\hline $\begin{array}{l}\text { Pissolato et al. (2016), } \\
\text { Brasil }\end{array}$ & $\begin{array}{l}\text { *10 puérperas a } \\
\text { amamentar } \\
\text { **Entender a influência, } \\
\text { na mãe, da } \\
\text { amamentação na } \\
\text { sexualidade do casal. }\end{array}$ & $\begin{array}{l}\text { A amamentação pode interferir na } \\
\text { intimidade do casal devido a } \\
\text { desconfortos, constrangimentos pelo } \\
\text { corpo e falta de tempo para o } \\
\text { companheiro. É necessário encarar a } \\
\text { sexualidade como uma necessidade } \\
\text { humana e a ela está associada à } \\
\text { preocupação pela contraceção. }\end{array}$ & $100 \%$ Alta \\
\hline
\end{tabular}

Da análise dos artigos, foram destacadas as necessidades e preocupações maternas, que posteriormente foram categorizadas consoante a temática, tendo emergido seis categorias de necessidades gerais (tema geral), as quais integram outras preocupações e necessidades (subtemas). De seguida realizou-se uma análise temática.

\subsection{Necessidade de Recuperação Física e Psicológica}

Os desconfortos físicos e o ganho ponderal preocupam as mães. Desejam regressar à forma física anterior à gravidez, não sendo isso uma prioridade. Reconhecem a importância de se alimentarem adequadamente pela qualidade do leite, apesar da preocupação com o peso (Carvalho et al., 2017; Xiao, Loke et al., 2020; Xiao, Ngai et al., 2020). As necessidades maternas são secundárias face às do RN (Kurth et al., 2016).

Preocupam-se com a sua nova imagem, podendo não se reconhecer no seu corpo pósparto (Carvalho et al., 2017). A cicatriz de episiotomia pode exacerbar esta insatisfação com a autoimagem (Marambaia et al., 2020). O cansaço, a privação de sono, a falta de tempo para si próprias contribui para diminuir a autoestima (Carvalho et al., 2017) e pode levar a sintomas depressivos (Kurth et al., 2016). O estudo de Iwata \& Tamakoshi (2018) corroborando estes achados reflete a influência negativa da fadiga materna na sensação de bem-estar materno, na relação mãe-filho e na relação e comunicação com os outros.

Há necessidade de realizar atividades fora do âmbito da maternidade (Albanese et al., 2020; Slomian et al., 2017). No entanto, quando o fazem sem os filhos, sentem-se culpadas (Albanese et al., 2020). 


\subsection{Necessidade de Desenvolvimento de Habilidades/Sensação de Controlo e Confiança no Cuidar do RN}

Uma das principais fontes de preocupação identificadas, relacionada com o RN, é a amamentação (Carvalho et al., 2017; Hannan et al., 2016; Henshaw et al., 2018; Kurth et al., 2016; Masala-Chokwe \& Ramukumba, 2017; Rouhi et al., 2019; Shorey \& Ng, 2019; Xiao, Loke et al., 2020). As mães consideram-se competentes no cuidar do RN se o conseguirem amamentar (Xiao, Loke et al., 2020). Se essa experiência não for positiva, traz sentimentos de culpa (Xiao, Loke et al., 2020). A falta de apoio dos profissionais, o incentivo da família para a introdução de leite de fórmula, a presença desconfortos ao amamentar levam ao abandono da amamentação (Xiao, Loke et al., 2020).

São também fontes de preocupação: o sono e o choro do RN, como aliviar cólicas abdominais, saber a temperatura ideal do ambiente ou da água do banho, a hidratação e alterações cutâneas do RN, reconhecer sinais ou sintomas de doença, a segurança do RN (Carvalho et al., 2017; Hannan et al., 2016; Nan et al., 2020; Xiao, Ngai et al., 2020) e a introdução de novos alimentos (Carvalho et al., 2017).

Ainda que as mães saibam que algumas características dos seus filhos, positivas (temperamento tranquilo) ou negativas (choro prolongado) não resultam das suas ações, tendem a interpretar as negativas como resultado disso, podendo levar a sentimentos de culpa ou depressão (Albanese et al., 2020).

\subsection{Necessidade de Informação}

As mães valorizam o acesso à informação, no entanto, o modo como a procuram difere. Umas recorrem a fontes formais de conhecimento, enquanto outras preferem o conhecimento que advém da experiência de outras mães (Albanese et al., 2020), fonte de apoio emocional, em quem confiam, e se sentem compreendidas, podendo trocar experiências, certificar-se das suas habilidades, comparar a evolução da criança, e assegurarem-se de que o que estão a experienciar é normal e comum (Nan et al., 2020; Slomian et al., 2017). Quando o tema é sexualidade, as mães podem sentir-se mais confortáveis com irmãs ou primas, elementos femininos da família, que as compreendam mantendo a confidencialidade (Cantarino et al., 2016).

As mães nem sempre recorrem primeiramente aos profissionais de saúde, por desvalorizarem os seus próprios problemas, por se sentirem incompreendidas, ou para evitar mostrar vulnerabilidade (Nan et al., 2020). O estigma e o medo de julgamentos inibem-nas de procurar ajuda, na área da saúde mental (Henshaw et al., 2018).

Preocupam-nas as diferentes recomendações prestadas pelos profissionais de saúde de obstetrícia e pediatria (Farewell et al., 2020; Kurth et al., 2016; Olander et al., 2019), principalmente sobre determinados assuntos menos frequentes do dia-a-dia, colocando em causa a confiança nos profissionais.

Recorrem à internet, não enquanto fonte de informação fidedigna, pois é cómodo e rápido (Albanese et al., 2020; Kurth et al., 2016; Nan et al., 2020; Rouhi et al., 2019; Shorey \& $\mathrm{Ng}, 2019$; Slomian et al., 2017).

Para se sentirem apoiadas sugerem o desenvolvimento de tecnologia e o recurso à teleconferência e programas de intervenção por pares que parecem ser uma mais-valia (Shorey \& Ng, 2019). Também sugerem o desenvolvimento de programas de cuidados pós-parto e o desenvolvimento de linhas telefónicas ou plataformas online de apoio, moderada por profissionais de saúde (Kurth et al., 2016; Xiao, Ngai et al., 2020). 


\subsection{Necessidade de Vida Conjugal e Sexual Satisfatórias}

Existem inúmeras preocupações que interferem na vida sexual, no PPP, nomeadamente a falta de tempo, a redução da líbido, a diminuição da lubrificação, a falta de interesse no parceiro e as mudanças físicas do corpo materno. Amamentar pode interferir na intimidade do casal, pela sensibilidade aumentada nos mamilos, pela presença do RN no quarto do casal e por poder ocorrer libertação de leite durante a relação sexual. Há uma diferenciação entre as mamas da mãe (fonte de alimento) das mamas de mulher (fonte de prazer) (Pissolato et al., 2016).

Nesta fase, existe medo de voltar a engravidar pelo que reiniciam a vida sexual e a contraceção simultaneamente (Pissolato et al., 2016). Outros autores como Caetano et al., (2018) referem que ocorre uma diminuição das relações sexuais e surgem preocupações relativamente ao planeamento familiar.

As mães referem fatores como a dor, o desconforto, o medo, a insatisfação com o seu corpo e a diminuição de desejo sexual como motivos para atrasarem o reinício da atividade sexual ou diminuírem a sua frequência (Cantarino et al., 2016; Carvalho et al., 2017; Marambaia et al., 2020).Estas têm de aprender a lidar com a sua nova imagem corporal, que as insatisfaz, ao mesmo tempo que desejam a sua aceitação, pelo companheiro (Cantarino et al., 2016), facto corroborado por outros autores como Caetano et al. (2018) Por outro lado, sentem ter menos tempo para o marido e para a relação (Pissolato et al., 2016).

Também sentem que não há uma verdadeira assunção da responsabilidade pelos companheiros, nem em cuidar do RN (Xiao, Ngai et al., 2020), nem na partilha de tarefas domésticas (Carvalho et al., 2017). Outros autores referem que esse facto e a iniquidade dos papéis sociais (que imputa maioritariamente à mãe a responsabilidade de cuidar do RN) levam a uma diminuição da satisfação com a relação conjugal (Graça et al., 2011; Lévesque et al., 2020).

A própria mãe aceita que é ela quem tem mais tempo para cuidar do filho (ao contrário do pai, que trabalha), no entanto, manifesta o desejo de poder compartilhar, mais equitativamente, os deveres com o parceiro (Carvalho et al., 2017; Xiao, Ngai et al., 2020). Este facto é corroborado por Graça et al. (2011) e Lévesque et al (2020). Os últimos reforçam que esta imposição de ser a mãe a cuidar do RN se mantém quando esta regressa ao trabalho, ficando em igualdade de circunstâncias com o companheiro.

\subsection{Necessidade de Apoio Familiar e Social}

O suporte recebido (emocional, instrumental, financeiro) é o pilar do bem-estar materno (Albanese et al., 2020). Realizar tarefas domésticas, ir às compras ou a consultas é difícil de concretizar sendo o apoio do companheiro e da família extremamente importantes (Kurth et al., 2016; Xiao, Ngai et al., 2020).

É interessante verificar que, apesar de poderem sentir que necessitam de apoio, algumas mães evitam pedi-lo por medo de demonstrar falhas no seu papel (Kurth et al., 2016), por não confiarem nos outros para cuidar dos filhos (por conceções de cuidar desatualizadas) (Xiao, Ngai et al., 2020) ou por receio de perderem autonomia por apoio demasiado invasivo (Faria-Schützer et al., 2020).

Em época de pandemia, ocorre um isolamento social das puérperas, privando-as do suporte que poderiam ter. Este facto tem implicações negativas na saúde mental, podendo também aumentar a ocorrência de violência doméstica (Farewell et al., 2020).

Regressar ao trabalho pode ser importante para aproximar as mães da identidade que tinham antes de engravidar (Albanese et al., 2020), no entanto outros autores salientam que isso exacerba a exigência de que estas devem ser capazes de cuidar dos filhos, manter a sua atividade profissional, não descurando as suas relações conjugais (Lévesque et al., 2020). É um período propício à suspensão da amamentação ou à introdução de leite adaptado. 
As mulheres são desencorajadas a amamentar: extrair o leite durante o horário de trabalho requer tempo, espaço adequado, com condições para preservar o leite, expondo as mães a julgamentos e críticas (Zhang et al, 2018).

\subsection{Necessidade de Apoio dos profissionais de saúde}

As mães queixam-se que o foco dos profissionais de saúde é o RN (Henshaw et al., 2018; Rouhi et al., 2019; Slomian et al., 2017) e que, quando são alvo de atenção, estes focamse na recuperação física, e raramente nas necessidades psicológicas e de informação (Nan et al., 2020). Outros autores salientam que os cuidados de saúde na maternidade estão centrado na gravidez, havendo uma diminuição considerável dos mesmos no PPP (Ribeiro et al., 2019) e que as mães expressam o desagrado por esse facto (Finlayson et al., 2020).

As mães querem ser questionadas sobre como estão ou se precisam de algo, em vez de serem elas a verbalizar. É necessário transmitir às mães que são também responsáveis pelo seu processo de recuperação (Corrêa et al., 2017). Algumas confessam vergonha em colocar questões (Xiao, Ngai et al., 2020).

Sugerem a existência de um profissional de referência para a gravidez e PPP (Slomian et al., 2017) e a realização de VD, pois sentem que o internamento no PPP foi curto, que as consultas foram superficiais e rápidas e ser difícil sair de casa com o filho, para recorrer aos serviços de saúde (Corrêa et al., 2017; Kurth et al., 2016; Nan et al., 2020; Xiao, Loke et al., 2020; Xiao, Ngai et al., 2020).

As mães gostariam que ensinassem aos companheiros habilidades para cuidar do RN e, aos avós, as recomendações mais atuais sobre o PPP e sobre o RN, de modo a que estes as apoiassem mais, diminuindo tensões intrafamiliares e intergeracionais (Xiao, Ngai et al., 2020).

\section{Conclusões}

Esta RSL apresenta algumas limitações pois as amostras dos estudos incluídos são reduzidas. Por outro lado, a diversidade dos estudos não permite realizar generalizações devido às diferentes políticas de apoio à maternidade e idade de reforma dos diferentes países, que influenciam a vivência deste período, bem como o apoio recebido pelos avós. Sugere-se investigar mais as vivências maternas e também paternas, neste período, no contexto português.

Esta RSL, tendo recorrido à síntese narrativa, permitiu clarificar e desocultar as vivências e comportamentos maternos no PPP, de forma abrangente, importante para prestar um cuidado mais efetivo e adequado às necessidades sentidas.

As mães necessitam de apoio na sua recuperação física e também psicológica, no desenvolvimento de habilidades para cuidar do RN, e de acesso a informação segura quando dela necessitam. Necessitam também do apoio do companheiro, e querem uma vida conjugal e sexual satisfatórias. $O$ apoio familiar e social é determinante para a mãe, se não for demasiado invasivo. Necessitam ainda de apoio dos profissionais de saúde para promover uma transição para a parentalidade mais serena, através da educação, esclarecimentos e vigilância de saúde. Achados que coincidem com a literatura existente.

Recomenda-se que o EEESMO diagnostique as preocupações maternas (atendendo a que sentimentos de culpa ou vergonha podem impedir as mães de verbalizar o que sentem) e desenvolva planos de cuidados personalizados, centrados na mãe/casal/família; que melhore os programas de preparação para o nascimento, para que no PPP, as expectativas maternas não sejam defraudadas pela realidade e implemente programas de recuperação pós-parto e VD. 


\section{Referências}

Albanese, A. M., Geller, P. A., Steinkamp, J. M., \& Barkin, J. L. (2020). In their own words: A qualitative investigation of the factors influencing maternal postpartum functioning in the united states. International Journal of Environmental Research and Public Health, 17(17), 128. https://doi.org/10.3390/ijerph17176021

Almalik, M. (2017). Understanding maternal postpartum needs : A descriptive survey of current maternal health services. Journal of Clinical Nursing, 26(23-24), 4654-4663. https://doi.org/10.1111/jocn.13812

American College of Obstetricians and Gynecologists. (2018). Committee Opinion No. 736: Optimizing postpartum care. Obstetrics and Gynecology, 131(5), e140-e150. https://doi.org/10.1097/AOG.0000000000002633

Aromataris, E., \& Munn, Z. (ed) (2020). JBI Manual for Evidence Synthesis. JBI Manual for Evidence Synthesis. https://doi.org/10.46658/jbimes-20-01

Caetano, A., Mendes, I., \& Rebelo, Z. (2018). Maternal concerns in the postpartum period: an integrative review. Revista de Enfermagem Referência, IV Série(17), 149-160. https://doi.org/10.12707/riv17074

Cantarino, S., Matas, M., Velasco, Abellan;Campos, S., Santos, A., \& Fernandez, I. (2016). Vivencias, experiencias y diferencias sexuales: mujer puérpera Española e inmigrante. Área sanitaria Palma de Mallorca (España). Enfermagem, IV(9), 115-123. http://www.scielo.mec.pt/pdf/ref/vserlVn9/serlVn9a13.pdf

Carvalho, J., Gaspar, M., \& Cardoso, A. (2017). Challenges of motherhood in the voice of primiparous mothers: Initial difficulties. Investigacion y Educacion En Enfermeria, 35(3), 285294. https://doi.org/10.17533/udea.iee.v35n3a05

Corrêa, M., Feliciano, K., Pedrosa, E. N., \& Souza, A. (2017). Acolhimento no cuidado à saúde da mulher no puerpério. Cadernos de Saude Publica, 33(3), 1-12. https://doi.org/10.1590/0102$311 \times 00136215$

Farewell, C. V., Jewell, J., Walls, J., \& Leiferman, J. A. (2020). A mixed-methods pilot study of perinatal risk and resilience during COVID-19. Journal of Primary Care and Community Health, 11, 1-8. https://doi.org/10.1177/2150132720944074

Faria-Schützer, D. B., Surita, F. G., Rodrigues, L., Paulino, D. S. D. M., \& Turato, E. R. (2020). Selfcare and health care in postpartum women with obesity: a qualitative study. Revista Brasileira de Ginecologia e Obstetricia, 42(1), 19-25. https://doi.org/10.1055/s-0039-3400456

Finlayson, K., Crossland, N., Bonet, M., \& Downe, S. (2020). What matters to women in the postnatal period: A meta-synthesis of qualitative studies. PLOS ONE, 15(4), 1-24. https://doi.org/10.1371/journal.pone.0231415

Graça, L., Figueiredo, M., \& Carreira, M. (2011). Contributos da intervenção de enfermagem de Cuidados de Saúde Primários para a transição para a maternidade. Revista de Enfermagem Referência, III Série(nº 4), 27-35. https://doi.org/10.12707/riii1012

Hannan, J., Brooten, D., Youngblut, J. A. M., \& Galindo, A. M. (2016). Comparing mothers' postpartum concerns in two clinical trials 18 years apart. Journal of the American Association of Nurse Practitioners, 28(11), 604-611. https://doi.org/10.1002/2327-6924.12384

Henshaw, E. J., Cooper, M. A., Jaramillo, M., Lamp, J. M., Jones, A. L., \& Wood, T. L. (2018). "Trying to figure out ff you're doing things right, and where to get the info": parents recall information and support needed during the first 6 weeks postpartum. Maternal and Child Health Journal, 22(11), 1668-1675. https://doi.org/10.1007/s10995-018-2565-3

Iwata, H., \& Tamakoshi, K. (2018). Course of maternal fatigue and its associated factors during the first 6 months postpartum : a prospective cohort study. January, 186-196. https://doi.org/10.1002/nop2.130

Kurth, E., Krähenbühl, K., Eicher, M., Rodmann, S., Fölmli, L., Conzelmann, C., \& Zemp, E. (2016). Safe start at home: What parents of newborns need after early discharge from hospital - A focus group study. BMC Health Services Research, 16(1), 1-14. https://doi.org/10.1186/s12913-016-1300-2 
Lévesque, S., Bisson, V., Charton, L., \& Fernet, M. (2020). Parenting and relational well-being during the transition to parenthood: challenges for first-time parents. Journal of Child and Family Studies, 29(7), 1938-1956. https://doi.org/10.1007/s10826-020-01727-z

Marambaia, C. G., Vieira, B. D. G., Alves, V. H., Rodrigues, D. P., Almeida, V. L. M., \& Calvão, T. F. (2020). Sexuality of women in the postpartum period: Reflexes of episiotomy. Cogitare Enfermagem, 25, 1-10. https://doi.org/10.5380/ce.v25i0.67195

Masala-Chokwe, M. E. T., \& Ramukumba, T. S. (2017). The lived experiences and social support needs of first-time mothers at health care facilities in the City of Tshwane, South Africa. Curationis, 40(1), 1-8. https://doi.org/10.4102/curationis.v40i1.1680

Mercer, R. T. (2004). Becoming a mother versus maternal role attainment. Journal of Nursing Scholarship, 36(3), 226-232. https://doi.org/10.1111/j.1547-5069.2004.04042.x

Nan, Y., Zhang, J., Nisar, A., Huo, L., Yang, L., Yin, J., Wang, D., Rahman, A., Gao, Y., \& Li, X. (2020). Professional support during the postpartum period: Primiparous mothers' views on professional services and their expectations, and barriers to utilizing professional help. BMC Pregnancy and Childbirth, 20(1), 1-13. https://doi.org/10.1186/s12884-020-03087-4

Negron, R., Martin, A., Almog, M., Balbierz, A., \& Howell, E. A. (2013). Social support during the postpartum period: Mothers' views on needs, expectations, and mobilization of support. Maternal and Child Health Journal, 17(4), 616-623. https://doi.org/10.1007/s10995-012-10374

Olander, E. K., Aquino, M. R. J. (Ryc), Chhoa, C., Harris, E., Lee, S., \& Bryar, R. M. (2019). Women's views of continuity of information provided during and after pregnancy: A qualitative interview study. Health and Social Care in the Community, 27(5), 1214-1223. https://doi.org/10.1111/hsc.12764

Ordem dos Enfermeiros. (2019). Regulamento das competências específicas do enfermeiro especialista em enfermagem de saúde materna e obstétrica. Diário Da República, 2. ${ }^{a}$ Série N. ${ }^{\circ} 85-3$ de Maio de 2019. https://dre.pt/application/conteudo/122216892

Pissolato, L., Alves, C., Prates, L., Wilhelm, L., \& Ressel, L. (2016). Breastfeeding and sexuality: an interface in the experience of puerperium. 4674-4680. https://doi.org/10.9789/21755361

Ribeiro, J., Lima, F., Soares, T., Oliveira, B., Klemtz, F., Lopes, K., \& Hartmann, M. (2019). Necessidades sentidas pelas mulheres no período puerperal. Revista de Enfermagem UFPE on Line, 13(1), 61-69. https://doi.org/10.5205/1981-8963-v13i1a235022p61-69-2019

Rouhi, M., Stirling, C. M., \& Crisp, E. P. (2019). Mothers' views of health problems in the 12 months after childbirth: A concept mapping study. Journal of Advanced Nursing, 75(12), 3702-3714. https://doi.org/10.1111/jan.14187

Shorey, S., \& Ng, E. D. (2019). Evaluation of a technology-based peer-support intervention program for preventing postnatal depression (Part 2): Qualitative study. Journal of Medical Internet Research, 21(8), 1-8. https://doi.org/10.2196/12915

Slomian, J., Emonts, P., Vigneron, L., Acconcia, A., Glowacz, F., Reginster, J. Y., Oumourgh, M., \& Bruyère, O. (2017). Identifying maternal needs following childbirth: A qualitative study among mothers, fathers and professionals. BMC Pregnancy and Childbirth, 17(1), 1-13. https://doi.org/10.1186/s12884-017-1398-1

Xiao, X., Loke, A., Zhu, S., Gong, L., Shi, H., \& Ngai, F. (2020). "The sweet and the bitter": Mothers' experiences of breastfeeding in the early postpartum period: A qualitative exploratory study in China. International Breastfeeding Journal, 15(1), 1-11. https://doi.org/10.1186/s13006-02000256-1

Xiao, X., Ngai, F., Zhu, S., \& Loke, A. (2020). The experiences of early postpartum Shenzhen mothers and their need for home visit services : a qualitative exploratory study. BMC Pregnancy and Childbirth, 20,1-13. https://doi.org/10.1186/s12884-019-2686-8

Yamada, R., Rasmussen, K. M., \& Felice, J. P. (2019). “What is 'enough,' and how do i Make It?”: a qualitative examination of questions mothers ask on social media about pumping and providing an adequate amount of milk for their infants. Breastfeeding Medicine, 14(1), 17-21. https://doi.org/10.1089/bfm.2018.0154

Zhang, Y., Jin, Y., Vereijken, C., Stahl, B., \& Jiang, H. (2018). Breastfeeding experience, challenges and service demands among Chinese mothers: A qualitative study in two cities. Appetite, 128(138), 263-270. https://doi.org/10.1016/j.appet.2018.06.027 